\title{
On the power-free parts of consecutive integers
}

\author{
by \\ B. M. M. DE Weger (Krimpen aan den IJssel) and \\ C. E. VAn de Woestijne (Leiden)
}

1. Introduction and main results. Considering the consecutive integers $48,49,50$, we observe that they are divisible by 16,49 , and 25 , respectively, and thus their squarefree parts are relatively small. However, as we consider larger integers, such striking examples are rare - exceptions are pairs $\left(x^{2}, d y^{2}\right)$ which satisfy Pell's equation for some squarefree $d \in \mathbb{Z}$ (see Lemma 2.1 below).

Let $n \geq 2$ and $k \geq 2$ be given integers. For any $m \in \mathbb{N}$, we denote the $k$ th power-free part of $m$ by $\omega_{k}(m)$, and define the number $\lambda_{n, k}(m)$ as

$$
\lambda_{n, k}(m)=\max _{i=0,1, \ldots, n-1} \omega_{k}(m-i) .
$$

For example, $\lambda_{3,2}(50)=3$.

It is immediate from this definition that $\lambda_{n, k}(m) \leq m$ for all $m \in \mathbb{N}$, and that this bound is attained infinitely often-for example, if $m$ is squarefree. Thus there are no nontrivial upper bounds to $\lambda_{n, k}(m)$. However, it is not obvious to see how small $\lambda_{n, k}(m)$ can be with respect to $m$. In this work, we construct families of $m$ with "small" $\lambda_{n, k}(m)$; furthermore, we develop some uniform lower bounds for this function, one of which follows from the $a b c$-conjecture. (We refer to [8] for an investigation of sets of almost powers for which the power-free parts are fixed.)

To investigate the problem outlined above, we try to find pairs $(e, c)$, with $e \geq 0$ and $c>0$, such that, for some particular $n$ and $k$,

$$
\liminf _{m \rightarrow \infty} \frac{\lambda_{n, k}(m)}{m^{e}} \leq c .
$$

To show the existence of such numbers, we begin with remarking that (2) is trivially satisfied by the pair $(1,1)$ for all $n$ and $k$, as $\omega_{k}(m) \leq m$ for all $m \in \mathbb{N}$. We may prove (2) for a smaller value of $e$ by constructing a sequence

1991 Mathematics Subject Classification: Primary 11N37; Secondary 11A05. 
of distinct positive integers $\left(m_{i}\right)_{i=1}^{\infty}$ such that $\lambda_{n, k}\left(m_{i}\right) \leq c m_{i}^{e}$ for all $i \geq 1$ and some constant $c>0$.

Following these lines, we prove

TheOrem 1.1. (i) Let $n \geq 2$ and $k \geq 2$. Then (2) is satisfied by $e=$ $1-k /(n k-1)$ and some effectively computable constant $c=c(n, k)$.

(ii) If $n$ and $k$ are odd, we may take $e=1-k /(n k-2)$.

(iii) If $n=k=2$, we have (2) for $e=0$ and $c=2$.

Regarding the question whether these upper bounds for $e$ are sharp, we begin with the following theorem of J. Turk [7, Theorem 3], which was proved using the theory of linear forms in logarithms, and as such, is effective.

Theorem 1.2 (Turk). Let $n \geq 2$ and $k \geq 3$. For all sufficiently large $m \in \mathbb{N}$, we have

$$
\lambda_{n, k}(m)>e^{(\log \log m)^{0.2}} .
$$

For $n \geq 3$ and $k=2$, we have

$$
\lambda_{n, k}(m)>(\log m)^{0.04} .
$$

Using estimates for the size of solutions of Thue equations, we have proved

TheOREM 1.3. Let $n \geq 2$ and $k \geq 3$. There exists a (large) effectively computable constant $c>0$ such that, for all $m \in \mathbb{N}$,

$$
\lambda_{n, k}(m)>c(\log m)^{1 /(2 k-1)} .
$$

We have not been able to obtain an absolute lower bound on $\lambda_{n, k}(m)$ that is polynomial in $m$. However, the following result, almost exactly the desired one, follows if we assume that the $a b c$-conjecture is true.

THEOREM 1.4. Let $n \geq 2$ and $k \geq 2$. If the abc-conjecture is true, (2) can be satisfied only if $e \geq 1-k /(n k-n)$.

Acknowledgements. We would like to thank R. Tijdeman and the referee for their many valuable comments, and F. Göbel for suggesting the problem.

2. Proof of Theorem 1.1. As we have remarked earlier, we may take $e=1$ for any $n$ and $k$. However, we can establish better solutions $e$ to (2) by constructing increasing sequences of positive integers $\left(m_{i}\right)_{i=1}^{\infty}$ such that $\lambda_{n, k}\left(m_{i}\right) \leq c m_{i}^{e}$ for all $i \geq 1$ and some constant $c>0$. This task is easy in case $n=k=2$.

LEMMA 2.1. If $n=k=2$, (2) holds with $e=0$ and $c=2$. 
Proof. As the Pell equation $x^{2}-2 y^{2}=1$ has infinitely many integral solutions $(x, y)$, we find an infinitude of integers $m>0$ such that $\lambda_{2,2}(m)$ $=2$.

This lemma proves Theorem 1.1(iii). For general $n$ and $k$, the construction of sequences $\left(m_{i}\right)_{i=1}^{\infty}$ starts with the consideration of systems of linear congruences of the following form:

$$
\left\{\begin{array}{l}
m_{i} \equiv b_{1}\left(\bmod c_{1}(i)^{k}\right) \\
m_{i} \equiv b_{2}\left(\bmod c_{2}(i)^{k}\right) \\
\vdots \\
m_{i} \equiv b_{n}\left(\bmod c_{n}(i)^{k}\right)
\end{array}\right.
$$

where $b_{1}, \ldots, b_{n}$ are a fixed sequence of consecutive integers, and the moduli $c_{1}^{k}, \ldots, c_{n}^{k}$ are positive integers, depending on $i$. Now if $m_{i}$ satisfies a system of this form for all $i$, we have

$$
\lambda_{n, k}\left(m_{i}\right) \leq \frac{m_{i}-b_{1}}{\min _{j=1, \ldots, n} c_{j}(i)^{k}} .
$$

Furthermore, using the Chinese Remainder Theorem and the fact that not all moduli are zero, it follows that we may assume

$$
0<m_{i}<\operatorname{lcm}\left(c_{1}(i)^{k}, \ldots, c_{n}(i)^{k}\right) \leq\left(c_{1}(i) \ldots c_{n}(i)\right)^{k} .
$$

To construct our actual $m_{i}$, we resort to polynomial methods: from now on, we will assume that the $c_{j}$ are given as functions of $i$ by polynomials with integer coefficients.

We will make use of both assertion and proof of the Chinese Remainder Theorem for polynomials, which we adapt from the integer version given by Cohen [3, Corollary 1.3.10]. Furthermore, we need a little symmetry result.

Lemma 2.2. Let $c_{1}(x), \ldots, c_{n}(x)$ be mutually relatively prime polynomials over a field $\mathbb{K}$, and let $b_{1}(x), \ldots, b_{n}(x)$ be arbitrary polynomials over $\mathbb{K}$. Then there exists a polynomial $f(x) \in \mathbb{K}$, with degree less than $\sum_{j=1}^{n} \operatorname{deg} c_{j}$, which satisfies

$$
\left\{\begin{array}{l}
f(x) \equiv b_{1}(x)\left(\bmod c_{1}(x)\right) \\
f(x) \equiv b_{2}(x)\left(\bmod c_{2}(x)\right) \\
\vdots \\
f(x) \equiv b_{n}(x)\left(\bmod c_{n}(x)\right)
\end{array}\right.
$$

and which is unique modulo $\prod_{j=1}^{n} c_{j}(x)$.

Proof. Put $M_{j}(x)=\prod_{l=1, l \neq j}^{n} c_{l}(x)$, and let $y_{j}(x)$ be the polynomial inverse of $M_{j}(x)$ modulo $c_{j}(x)$. Put $f=\sum_{j=1}^{n} b_{j} y_{j} M_{j} \bmod \prod_{j=1}^{n} c_{j}$. Then $f(x)$ satisfies our claims, which may be verified in a straightforward manner. 
LEMma 2.3. Let $f(x)$ be any polynomial over a field $\mathbb{K}$ such that it has a polynomial inverse $g(x)$ modulo $x^{m+1}$ for some nonnegative integer $m$. Then $f(-x) g(-x) \equiv 1\left(\bmod x^{m+1}\right)$ as well.

Pro of. If $x^{m+1} \mid f(x) g(x)-1$, then $(-x)^{m+1} \mid f(-x) g(-x)-1$.

Proof of Theorem 1.1(i) and (ii). We first prove the case when $n$ is odd. The even case will be analogous.

Consider the system

$$
\left\{\begin{array}{l}
m_{i} \equiv-\frac{n-1}{2}\left(\bmod \left(a i-\frac{n-1}{2}\right)^{k}\right), \\
m_{i} \equiv-\frac{n-3}{2}\left(\bmod \left(a i-\frac{n-3}{2}\right)^{k}\right), \\
\vdots \\
m_{i} \equiv \frac{n-1}{2}\left(\bmod \left(a i+\frac{n-1}{2}\right)^{k}\right),
\end{array}\right.
$$

where $a$ is an integer parameter which will be assigned a value later on. Thus we have $c_{j}(x)=a x+j$ in (3), letting our index $j$ run from $-(n-1) / 2$ to $(n-1) / 2$. Note that $m_{i} \equiv 0\left(\bmod (a i)^{k}\right)$.

Following the proof of Lemma 2.2, we write

$$
\text { (8) } \quad M_{j}(x)=\prod_{l=-(n-1) / 2, l \neq j}^{(n-1) / 2}(a x+l)^{k}=\prod_{l=-(n-1) / 2, l \neq j}^{(n-1) / 2}(a x+j+(l-j))^{k}
$$

as a polynomial $\sum_{l=0}^{(n-1) k} m_{j, l}(a x+j)^{l}$ in $a x+j$, and likewise $y_{j}(x)$, the polynomial inverse of $M_{j}(x)$, is written $\sum_{l=0}^{k-1} y_{j, l}(a x+j)^{l}$. As $M_{j} y_{j} \equiv 1$ $\left(\bmod (a x+j)^{k}\right)$, we can solve the coefficients $y_{j, l}$ from the linear systems

$$
\mathbf{A}_{j} \vec{y}_{j}:=\left[\begin{array}{cccc}
m_{j 0} & 0 & \ldots & 0 \\
m_{j 1} & m_{j 0} & \ddots & \vdots \\
\vdots & \vdots & \ddots & 0 \\
m_{j, k-1} & m_{j, k-2} & \ldots & m_{j 0}
\end{array}\right]\left(\begin{array}{c}
y_{j 0} \\
y_{j 1} \\
\vdots \\
y_{j, k-1}
\end{array}\right)=\left(\begin{array}{c}
1 \\
0 \\
\vdots \\
0
\end{array}\right),
$$

for $j=-(n-1) / 2, \ldots,(n-1) / 2$. We have $\operatorname{det} \mathbf{A}_{j}=m_{j 0}^{k}$; from (8), we have

$$
m_{j 0}= \pm((n-1) / 2+j) !^{k} \cdot((n-1) / 2-j) !^{k} .
$$

As the binomial coefficient $\left(\begin{array}{c}n-1 \\ (n-1) / 2+j\end{array}\right)$ is integral, we find that $m_{j 0} \mid(n-1) !^{k}$. Thus $(n-1) !^{k^{2}} y_{j}(x) \in \mathbb{Z}[x]$ for all $j$.

Let us denote by cont $P$ the content of a polynomial $P \in \mathbb{Z}[x]$, i.e. the greatest common divisor of its coefficients with respect to expansions in 
powers of $x$. We have

$$
f(x)=\sum_{j=-(n-1) / 2}^{(n-1) / 2} j y_{j}(x) M_{j}(x) .
$$

As $a^{k} \mid \operatorname{cont}\left(M_{j}\right)$ for $j \neq 0$, and $M_{0}$ cancels out, $f(x)$ has integer coefficients if we choose $a$ equal to $(n-1) !^{k}$.

It remains to investigate the quotients of $f(x)-j$ and $(a x+j)^{k}$, for $j=-(n-1) / 2,-(n-3) / 2, \ldots,(n-1) / 2$, which we know to be in $\mathbb{Q}[x]$. It is an implication of Gauss' Lemma that if $g, h \in \mathbb{Z}[x]$ with $g \mid h$, and $g$ has content one, then $h / g \in \mathbb{Z}[x]$. We may thus conclude that $f(x)-j$ is divisible over $\mathbb{Z}$ by $((a / j) x+1)^{k}$ if $j \neq 0$, and by $x^{k}$ if $j=0$, recalling that $j \mid a$ for all $j$ considered, and that the equations (6) remain valid over $\mathbb{Q}$ upon multiplying some $c_{j}(x)$ by a nonzero scalar.

We take our solution $m_{i}$ to be $f(i)$, so that

$$
\lambda_{n, k}\left(m_{i}+(n-1) / 2\right) \leq m_{i} / i^{k},
$$

an upper bound which is a polynomial in $i$ of degree $d \leq(n-1) k-1$. It follows that

$$
e=\frac{d}{d+k}=1-\frac{k}{d+k} \leq 1-\frac{k}{n k-1} .
$$

We can, in some cases, improve this bound as follows. Suppose $k$ is odd. It is not difficult to see that $M_{-j}(x)=M_{j}(-x)$. Thus by our second lemma, we also have $y_{-j}(x)=y_{j}(-x)$. We find that the coefficient of $(a x+j)^{n k-1}$ in $M_{-j} y_{-j}$ is

$$
m_{-j,(n-1) k} y_{-j, k-1}=(-1)^{n(k-1)} m_{j,(n-1) k} y_{j, k-1}=m_{j,(n-1) k} y_{j, k-1},
$$

as $k$ is odd. Thus the $(m k-1)$ th degree term of $j y_{j} M_{j}-j y_{-j} M_{-j}$ cancels out, yielding an upper bound for $\lambda_{n, k}\left(m_{i}\right)$ of polynomial degree $d \leq(n-1) k-2$ in $i$; and we find

$$
e=\frac{d}{d+k} \leq 1-\frac{k}{n k-2} .
$$

If $n \equiv 3(\bmod 4)$, it appears that our solution sequence $m_{i}$ consists of negative numbers; this can be amended by reversing the order of the moduli, or of the numbers $-(n-1) / 2,-(n-3) / 2, \ldots,(n-1) / 2$. This proves 1.1(ii).

Finally, when $n$ is even, we consider

$$
\begin{cases}m_{i} \equiv 0 & \left(\bmod (a i)^{k}\right), \\ m_{i} \equiv 1 & \left(\bmod (a i+1)^{k}\right), \\ \vdots & \\ m_{i} \equiv n-1 & \left(\bmod (a i+n-1)^{k}\right) .\end{cases}
$$


The proof runs entirely analogously, except that $j$ runs from 0 to $n-1$, and the symmetry considerations for odd $k$ cannot be extended to this case. Note that the same value for $a$ suffices to obtain integral solutions; we have $m_{j 0}= \pm j !^{k}(n-1-j) !^{k} \mid(n-1) !^{k}$.

It is possible to obtain explicit solutions to the "Chinese Remainder systems" considered in this proof. For example, when $n=3$ and $k=3$, taking $a=8$ gives $f(i)=3932160 i^{7}-172032 i^{5}+2240 i^{3}$. Consider $f(5)=$ 306662680000 . It is easily verified that $(8 \cdot 5-1)^{3}\left|f(5)-1,(8 \cdot 5+1)^{3}\right| f(5)+1$, and $5^{3} \mid f(5)$. To give another example, when $n=4$ and $k=2$, taking $a=36$ gives $f(i)=43535646720 i^{7}+12697896960 i^{6}+1434392064 i^{5}+78382080 i^{4}+$ $2086560 i^{3}+22680 i^{2}$. Here we find $f(5)=3604158765387000$, and thus $5^{2}\left|f(5),(36 \cdot 5+1)^{2}\right| f(5)-1,(18 \cdot 5+1)^{2} \mid f(5)-2$, and $(12 \cdot 5+1)^{2} \mid f(5)-3$.

It should be noted that the choice $a=(n-1) !^{k}$ is an upper bound, and is much too high in many concrete cases. For fixed $n$ and $k$, one may establish a tighter upper bound by reducing $\operatorname{det} \mathbf{A}_{j}$; this is possible because most equations are homogeneous. E.g., for $n=9$ and $k=4, a=5880=$ $2^{3} \cdot 3 \cdot 5 \cdot 7^{2}$ is enough to obtain an integral expression for $m_{i}$, whereas $8 !^{4}=$ 2642908293365760000 ; for $n=16$ and $k=4, a=360360=2^{3} \cdot 3^{2} \cdot 5 \cdot 7 \cdot 11 \cdot 13$ is sufficient, whereas $(16-1) !^{4} \approx 10^{24}$.

When evaluating a concrete case, we recommend to take as initial value for $a$ the product of all primes dividing $(n-1)$ !, and adding some factors as necessary (these may be derived from the denominators appearing in the output of the Chinese Remainder Theorem, and especially the denominator of the constant term).

3. Is our bound sharp? In the remainder of this work, we address some possible approaches to settle the question whether the constructed possible values for $e$ are optimal. The quoted result of Turk (Theorem 1.2), which provides a lower bound for $\lambda_{n, k}(m)$ that is polynomial in $\log m$, goes without further comments here.

3.1. The Thue equation: proof of Theorem 1.3. We can view the problem of finding $X \in \mathbb{N}$ where all of $X, X+1, \ldots, X+n-1$ contain reasonably large $k$ th power factors, as the study of the system of Thue equations

$$
\left\{\begin{array}{l}
a_{2} x_{2}^{k}-a_{1} x_{1}^{k}=1, \\
a_{3} x_{3}^{k}-a_{1} x_{1}^{k}=2, \\
\vdots \\
a_{n} x_{n}^{k}-a_{1} x_{1}^{k}=n-1
\end{array}\right.
$$

which is clearly solvable if we substitute the $k$ th power-free part of the 
consecutive integers $X, X+1, \ldots, X+n-1$ for the coefficients $a_{1}, \ldots, a_{n}$ (thus we have $X=a_{1} x_{1}^{k}$ ).

We will make use of a result of Bugeaud and Györy, which estimates the size of the solutions of a Thue equation, i.e. an equation of the form $F(x, y)=b$ in $x, y \in \mathbb{Z}$, where $F(x, y)$ is a bivariate polynomial of degree $k \geq 2$ with integer coefficients. Their theorem reads [2, Theorem 3]:

Lemma 3.1. Let $(x, y)$ be a solution of $F(x, y)=b$, as described above. Then

$$
\max \{|x|,|y|\}<\exp \left\{c_{4} H^{2 k-2}(\log H)^{2 k-1} \log B\right\},
$$

where $B \geq 3$ is an upper bound for $b, k \geq 3$ is the degree of the equation, $H \geq 3$ is an upper bound for the height of $F$, i.e. the maximum absolute value of its coefficients, and $c_{4}=c_{4}(k)=3^{3(k+9)} k^{18(k+1)}$.

Note that the case $k=2$ is not covered by this result.

Proof of Theorem 1.3. Assume that $n \geq 2$, take some $X \in \mathbb{N}, X>2$, and consider the equation

$$
a_{2} x_{2}^{k}-a_{1} x_{1}^{k}=1
$$

taking the coefficients $a_{2}$ and $a_{1}$ as defined above. Following Bugeaud and Györy's notations, we may take $B=3$. On putting $X=a_{1} x_{1}^{k}$ and $H=$ $\max \left\{3,\left|a_{2}\right|,\left|a_{1}\right|\right\}$, it follows that $\lambda_{n, k}(X) \geq H$, unless both coefficients are less than 3 in absolute value. However, this would constitute a nontrivial solution to the equation $\left|x^{k}-2 y^{k}\right|=1$, whereas theorems of Darmon and Merel [4] and Skolem [6, Theorem 7 of Chapter 23] show that this equation has no solutions with $|x y|>1$. Thus we have $\lambda_{n, k}(X) \geq H$ in all cases. We have

$$
\log X \leq \log \left(\max \left\{\left|x_{1}\right|,\left|x_{2}\right|\right\}^{k} H\right)<k c_{4} H^{2 k-2}(\log H)^{2 k-1} \log B+\log H
$$

so that, for some positive $c_{5}$,

$$
c_{5}(\log X)^{1 /(2 k-2)}<H(\log H)^{(2 k-1) /(2 k-2)} \leq\left(\frac{2 k-1}{e} H\right)^{(2 k-1) /(2 k-2)} .
$$

It is clear that these considerations imply our theorem.

3.2. The abc-conjecture: proof of Theorem 1.4. Finally, we will explore the consequences of the $a b c$-conjecture for the existence of consecutive integers with large perfect power divisors. We denote by $r(n)$ the radical of a number $n \in \mathbb{Z}$, i.e. the product of all distinct primes dividing $n$.

The $a b c$-conjecture, stated by J. Oesterlé and D. Masser, runs as follows $[1,5]$ :

Conjecture 3.2. For all $\varepsilon>0$, there exists a positive constant $C_{\varepsilon}$ such that for any integers $a, b, c>0$ satisfying $a+b=c$ and $\operatorname{gcd}(a, b)=1$, we 
have

$$
r(a b c)>C_{\varepsilon} c^{1-\varepsilon} .
$$

This clearly implies that for any integers $0<x<y$ we have

$$
(y-x) r(x y)>C_{\varepsilon} y^{1-\varepsilon} .
$$

It is possible to apply this conjecture to our problem, with nearly optimal results, viz. Theorem 1.4 from the introduction, which we will prove as a corollary to the following proposition.

Proposition 3.3. Let $n$ and $k$ be integers, both greater than 2 , and let $\delta>0$. For any $X \in \mathbb{N}$ write

$$
X=a_{1} x_{1}^{k}, \quad X+1=a_{2} x_{2}^{k}, \ldots, X+(n-1)=a_{n} x_{n}^{k},
$$

with the $a_{i} k$-free. Then assuming the abc-conjecture, we have

$$
a_{1} \ldots a_{n}>X^{((n-1) k-n-\delta) /(k-1)}
$$

for all sufficiently large $X$.

For the proof we make use of a theorem of M. Langevin [5, Théorème 2], which we state as a lemma.

Lemma 3.4 (Langevin). Let $b_{1}<\ldots<b_{n}$ be integers and $\varepsilon>0$ in $\mathbb{R}$. The abc-conjecture implies

(10) $\quad r\left(\left(X+b_{1}\right) \ldots\left(X+b_{n}\right)\right)>X^{n-1-\varepsilon} \quad$ for all sufficiently large $X$.

P r o of (of Proposition 3.3). Take $n, k \in \mathbb{Z}$, both greater than 2, and take $\varepsilon>0$ small enough. Putting $b_{1}=0, \ldots, b_{n}=n-1$, we have, by Lemma 3.4,

$$
X^{n-1-\varepsilon}<r(X(X+1) \ldots(X+(n-1))) \leq\left(a_{1} \ldots a_{n}\right)\left(\frac{(X+n)^{n}}{a_{1} \ldots a_{n}}\right)^{1 / k},
$$

so that

$$
\left(a_{1} \ldots a_{n}\right)^{1-1 / k}>X^{n-n / k-1-\varepsilon}\left(1+\varepsilon^{\prime}\right)^{-n / k} \geq X^{n-n / k-1-\delta}
$$

for some $\varepsilon^{\prime}>0$. This proves the proposition.

Proof of Theorem 1.4. From our proposition, it follows that

$$
\max \left\{a_{1}, \ldots, a_{n}\right\}>X^{((n-1) k-n-\delta) /(n k-n)}
$$

for any $\delta>0$ and all sufficiently large $X \in \mathbb{N}$. Our theorem follows.

Browkin and Brzeziński have provided a generalization of the $a b c$-conjecture, which they call the $n$-conjecture (cf. [1]). There, they deal with $n$ integers summing up to zero, and the exponent $1-\varepsilon$ is replaced by $2 n-5-\varepsilon$. It might be interesting to see whether a generalization of Langevin's results to cover this more general case provides a better bound for $e$. 


\section{References}

[1] J. Browkin and J. Brzeziński, Some remarks on the abc-conjecture, Math. Comp. 62 (1994), 931-939.

[2] Y. Bugeaud and K. Györy, Bounds for the solutions of Thue-Mahler equations and norm form equations, Acta Arith. 74 (1996), 273-292.

[3] H. Cohen, A Course in Computational Algebraic Number Theory, 2nd ed., Springer, Berlin, 1995.

[4] H. Darmon and L. Merel, Winding quotients and some variants of Fermat's Last Theorem, J. Reine Angew. Math. 490 (1997), 81-100.

[5] M. Langevin, Cas d'égalité pour le Théorème de Mason et applications de la conjecture (abc), C. R. Acad. Sci. Paris Sér. I 317 (1993), 441-444.

[6] L. J. Mordell, Diophantine Equations, Academic Press, London, 1969, 207-208.

[7] J. Turk, Almost powers in short intervals, Arch. Math. (Basel) 43 (1984), 157-166.

[8] B. M. M. de Weger and C. E. van de Woestijne, On the diameter of sets of almost powers, Acta Arith., this issue, 371-385.

Sportsingel 30

2924 XN Krimpen aan den IJssel

The Netherlands

E-mail: deweger@xs4all.nl
Mathematical Institute

Universiteit Leiden P.O. Box 9512, 2300 RA Leiden The Netherlands E-mail: cvdwoest@wi.LeidenUniv.nl 